\title{
Resolution of Lung Injury after a Single Event of Aspiration
}

\section{A Model of Bilateral Instillation of Whole Gastric Fluid}

\author{
Joaquín D. Araos, * Pedro S. Ayala, * Manuel Meneses, ${ }^{\dagger}$ Rafael Contreras, * Andrea Cutiño, * Rebeca M. Montalva, * \\ Henry D. Tazelaar, ${ }^{\ddagger}$ and Gisella R. Borzone*
}

From the Department of Respiratory Diseases and Medical Research Center, * Pontificia Universidad Católica de Chile, Santiago, Chile; the Department of Pathology, ${ }^{\dagger}$ Instituto Nacional del Tórax, Santiago, Chile; and the Department of Pathology, ${ }^{\ddagger}$ Mayo Clinic, Scottsdale, Arizona

\author{
Accepted for publication \\ July 1, 2015. \\ Address correspondence to \\ Gisella R. Borzone, M.D., \\ Ph.D., Department of Respira- \\ tory Diseases, Pontificia Uni- \\ versidad Católica de Chile, \\ Marcoleta 350, Piso 1 Interior, \\ Santiago, Chile. E-mail: \\ gborzone@med.puc.cl.
}

\begin{abstract}
Gastric aspiration is a high-risk condition for lung injury. Consequences range from subclinical pneumonitis to respiratory failure, with fibrosis development in some patients. Little is known about how the lung repairs aspiration-induced injury. By using a rat model of single orotracheal instillation of whole gastric contents, we studied the time course of morphological and biochemical changes during injury and resolution, and evaluated whether repair involved long-term fibrosis. Anesthetized rats received one gastric fluid instillation. At 4, 12, and 24 hours and 4 and 7 days, we performed lung histological studies and biochemical measurements in bronchoalveolar lavage fluid and lung tissue. Physiological measurements were performed at 12 to 24 hours. Long-term outcome was studied histologically at day 60 . During the first 24 hours, severe peribronchiolar injury involving edema, intra-alveolar proteinaceous debris, hemorrhage, increased neutrophils and cytokines, and physiological dysfunction were observed. At days 4 and 7, an organizing pneumonia (OP) pattern developed, with foreign-body giant cells and granulomas. Lung matrix metalloproteinase 9 and 2 activities increased, with metalloproteinase- 9 linked to early inflammation and metalloproteinase-2 to OP. At day 60, lung architecture was normal. In conclusion, a continuum of alterations starting with severe injury, evolving toward $\mathrm{OP}$ and later resolving, characterizes the rat single aspiration event. In addition to identifying markers of staging and severity, this model reveals that OP participates in the repair of aspiration-induced injury. (Am J Pathol 2015, 185: 2698-2708; http://dx.doi.org/10.1016/j.ajpath.2015.07.001)
\end{abstract}

Aspiration of gastric contents into the lungs is common among patients with an impaired gag reflex, a depressed level of consciousness, drug use, and gastrointestinal disorders. ${ }^{1,2}$ Consequences of aspiration are variable, ranging from subclinical pneumonitis to severe respiratory failure. ${ }^{3}$

Several approaches have been used in animal models to gain insights into the pathogenesis of aspiration-induced lung injury. Instillation of individual components of gastric fluid has contributed to the understanding of their relative roles in lung injury. . $^{1-13}$ Thus, derangement of the alveolarcapillary barrier with edema and an intense inflammatory reaction occur with acid instillation, ${ }^{4-7}$ whereas a delayed inflammatory reaction, followed by granuloma formation without significant edema, results when acid-free gastric food particles are instilled. . $^{8,11}$ Synergistic effects are obtained when acid and gastric food particles are instilled in combination. ${ }^{1,8}$ Interestingly, few studies have used the whole gastric fluid to study the pathogenesis of aspiration. Those studies have used low volumes of gastric fluid instilled into small areas of the lung with the aim of answering questions mainly related to gastric aspiration and lung transplant rejection. $^{14-16}$

Although valuable to our understanding of the pathophysiology of aspiration-induced lung injury, existing

\footnotetext{
Supported by FONDECYT (National Fund for Science and Technology) grant 1120943 (G.R.B. and M.M.).

Disclosures: None declared.
} 


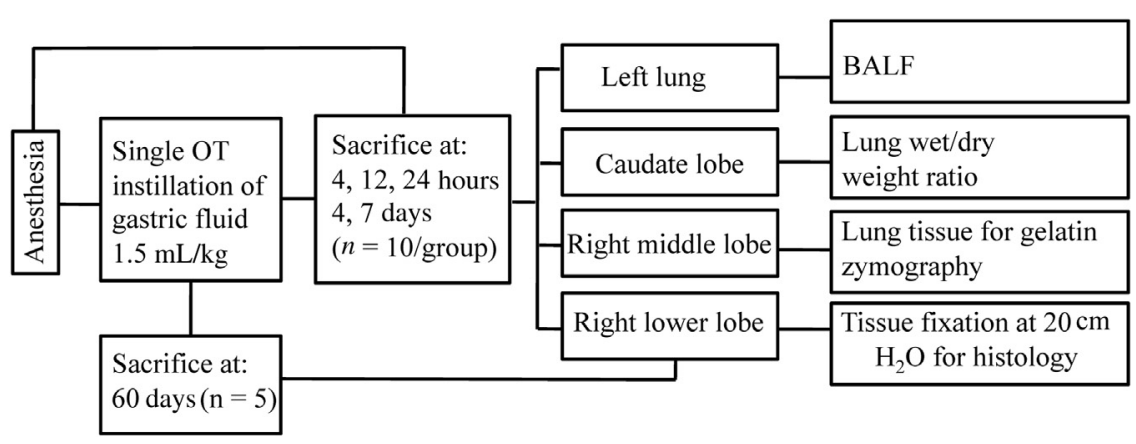

Figure 1 Diagram showing animal groups, tissue sampling, and analysis. BALF, bronchoalveolar lavage; OT, orotracheal.

models have not addressed the study of the continuum of changes after a single event of bilateral aspiration of whole gastric contents. This approach could help to understand what determines the histological pattern in human aspirationinduced acute lung injury (ALI), because either diffuse alveolar damage or organizing pneumonia (OP), the two main patterns described under the term ALI ${ }^{17}$ can be found in lung biopsy specimens from patients who have aspirated. ${ }^{18-20}$ Whether the OP pattern in this context represents a de novo process or, instead, originates from an earlier inflammatory reaction is unknown.

Moreover, it is still unknown how and to what extent the lung repairs in individuals who survive the early vascular and mechanical derangements induced by a single event of aspiration. In mice models of hydrochloric acid-induced ALI in which resolution of injury markers allowed for evaluation of subacute changes, fibrosis was found. ${ }^{4,7}$ However, it has not yet been answered whether this fibrosis is persistent (ie, pathological fibrosis) or, instead, is reversible as part of a normal healing process that ends with restoration of normal lung architecture.

We postulate that an animal model of bilateral instillation of a single dose of whole gastric fluid to study the time course of changes during ALI, its resolution and repair, could contribute to a better understanding of the full range of lung responses after aspiration in humans.

The purposes of this study, therefore, were as follows: First, to develop a rat model of single orotracheal instillation of whole gastric contents. Second, to characterize ALI, using a method recently published by the American Thoracic Society. ${ }^{21}$ Third, to evaluate key elements known to participate in lung injury resolution and repair but not included in American Thoracic Society guidelines, such as organization of the exudate, presence of myofibroblasts, collagen deposition, and activity of matrix metalloproteinases (MMPs). Finally, we studied histological changes at a later time to evaluate longterm consequences of a single instillation of gastric fluid.

Because lung biopsy specimens from patients represent a single snap-shot of the events that occur after aspiration, a study of early and late changes induced in this model will lead to a better understanding of the full range of lung responses, allow for the identification of markers of injury and repair likely to be evaluated in humans who aspirate, and improve treatment strategies.

\section{Materials and Methods}

\section{Model of Orotracheal Instillation of Rat Gastric Contents}

The study was performed according to a protocol submitted to and approved by the Animal Research Ethics Committee of the Pontificia Universidad Católica de Chile (Santiago, Chile).

\section{Gastric Contents Pool}

Adult male Sprague-Dawley rats (weight, $300 \pm 10 \mathrm{~g}$ ) fasted overnight were anesthetized i.p. with xylazineketamine ( 5.1 and $55.1 \mathrm{mg} / \mathrm{kg}$, respectively) to obtain gastric fluid through a gastrotomy. Gastric fluid samples were pooled, filtered through a $100-\mu \mathrm{m}$ mesh, and kept at $-80^{\circ} \mathrm{C}$. Animals were euthanized thereafter by exsanguination under anesthesia.

\section{Orotracheal Instillation}

Under the same anesthetic protocol, another set of animals was orotracheally intubated with a 22 gauge wire-fed catheter. Visualization of the glottis was achieved using a modified human otoscope (Welch Allyn, Skaneateles Falls, NY). A volume of gastric fluid previously determined by the authors (data not shown) to distribute evenly $(1.5 \mathrm{~mL} / \mathrm{kg}, \mathrm{pH}=1.69)$ was instilled, and animals were allowed to recover spontaneously from anesthesia.

\section{Study Groups}

Histological and biochemical studies were performed at 4, 12, and 24 hours, and 4 and 7 days after instillation $(n=10$ per group). Physiological measurements were performed in a small group of animals at 12 to 24 hours $(n=6)$ and at day $7(n=6)$.

Table 1 Acute Lung Injury Scoring System

\begin{tabular}{llll}
\hline & \multicolumn{3}{l}{ Score per field } \\
\cline { 2 - 4 } Parameter of acute lung injury & 0 & 1 & 2 \\
\hline Inflammatory cells in the alveolar space & 0 & $1-5$ & $>5$ \\
Inflammatory cells in the interstitial space & 0 & $1-5$ & $>5$ \\
Hyaline membranes & 0 & $1-10$ & $>10$ \\
Proteinaceous debris filling the airspaces & 0 & $1-10$ & $>10$ \\
Alveolar septal thickening & $<2 \times 2 \times$ to $4 \times>4 \times$ \\
\hline
\end{tabular}

Adapted and modified from Matute-Bello et al, ${ }^{21}$ with permission from the American Thoracic Society (copyright 2015). 


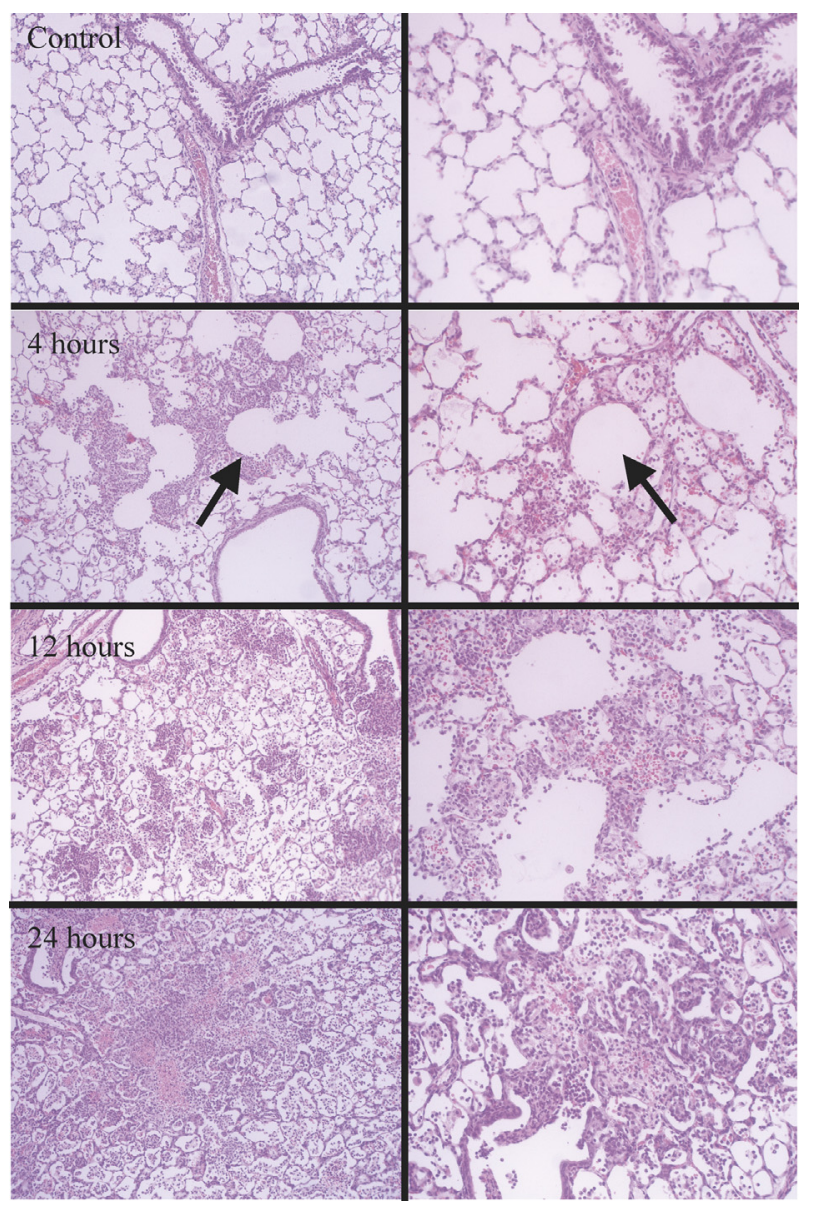

Figure 2 Histological evaluation of acute lung injury in the first 24 hours. Light microscopy (hematoxylin and eosin stain) of rat lung at 4, 12, and 24 hours after whole gastric content instillation. A cellular infiltrate of polymorphonuclear neutrophils and red blood cells with abundant intra-alveolar proteinaceous material in a peribronchiolar distribution (arrows) is seen at 4 hours. A more intense reaction is seen at 12 and 24 hours, with confluence of the inflammatory exudates between neighboring bronchioles at 24 hours. Original magnification: $\times 40$ (left panels); $\times 100$ (right panels).

To evaluate reversibility of histological changes, another set of animals $(n=5)$ was studied at day 60 after instillation. Animals without intervention served as controls because only a negligible change in the proportion of alveolar cells was seen in saline-treated animals.

\section{Sample Collection}

Lungs were excised en bloc, and the left main bronchus was cannulated for bronchoalveolar lavage (BAL). For each animal, three aliquots of $0.15 \mathrm{~mol} / \mathrm{L}$ saline $(1 \mathrm{~mL}$ each) were instilled, immediately aspirated, pooled, and stored at $-80^{\circ} \mathrm{C}$ after cell count. The right upper and middle lobes were excised and frozen for biochemical and molecular analysis. The caudate lobe was used to obtain the wet/dry weight ratio. The right lower lobe was fixed at $20 \mathrm{~cm} \mathrm{H}_{2} \mathrm{O}$ with $10 \%$ buffered formaldehyde solution and paraffin embedded for histological studies. At day 60, only histology was performed. Figure 1 illustrates the study protocol and tissue sampling diagram.
Evaluation of Lung Injury, Resolution, and Repair

Evaluation of ALI and Resolution according to American Thoracic Society Guidelines ${ }^{21}$

Histological evidence of tissue injury. For each animal, four whole lobe longitudinal sections ( $5 \mu \mathrm{m}$ thick) were obtained and stained with hematoxylin and eosin for analysis by light microscopy, and a mean score was obtained. A boardcertified pathologist (M.M.) scored samples according to reference, ${ }^{21}$ with modifications shown in Table 1 . To account for peribronchiolar distribution of injury, we measured the percentage of involved bronchioles.

Alterations of alveolar capillary barrier. Total protein concentration in BAL fluid (BALF) was measured using the Bradford assay. ${ }^{22}$

Concentration of high-molecular-weight proteins in BALF: SDS-PAGE of BALF was used to study changes in protein composition in comparison with plasma samples, using Coomassie Blue stain.

Lung wet/dry weight ratio of the caudate lobe was obtained using an oven at $60^{\circ} \mathrm{C}$ until stable dry weight was achieved.

Hemoglobin concentration in BALF was measured by light absorbance at 510- to $650-\mathrm{nm}$ wavelength using a spectrophotometer (Shimadzu, Kyoto, Japan).

Inflammatory response. BALF total and differential cell count was obtained using a Neubauer chamber and a cytospin slide centrifuge (StatSpin Cytofuge 2; Iris, Westwood, MA). Cytoslides were stained with DiffQuik (QCA, Tarragona, Spain).

BALF levels of IL- 6 , IL- $1 \beta$, tumor necrosis factor- $\alpha$, transforming growth factor- $\beta 1$, and monocyte chemoattractant protein-1 were measured in duplicate using available enzymelinked immunosorbent assay kits (Quantikine; R\&D Systems, Minneapolis, MN), according to manufacturer's instructions, and microplates were read using a microplate reader (BIO-TEK Instruments, Winooski, VT).

Gelatinolytic activity of lung tissue MMP-2 and MMP-9 was studied using zymography. ${ }^{23}$ Lung homogenate supernatant total protein $(30 \mu \mathrm{g})$ was placed into a gelatin-containing electrophoresis gel $(10 \%$ polyacrylamide and $1 \%$ gelatin under nonreducing conditions). After electrophoresis, gels were washed in 2.5\% Triton X-100 (Sigma-Aldrich, St. Louis, MO) to remove SDS, incubated overnight at $37^{\circ} \mathrm{C}$, and stained with $0.1 \%$ Coomassie Brilliant Blue. Densitometric analysis was performed using ImageJ software version 1.02 (NIH, Bethesda, MD).

Evidence of physiological dysfunction. End expiratory lung volume was measured ex vivo using the Archimedes principle, by subtracting the lung's weight from the volume of saline displaced (assuming tissue density equal to 1 ).

Lung compliance was measured ex vivo after a volume history was obtained, by sequentially inflating and deflating 


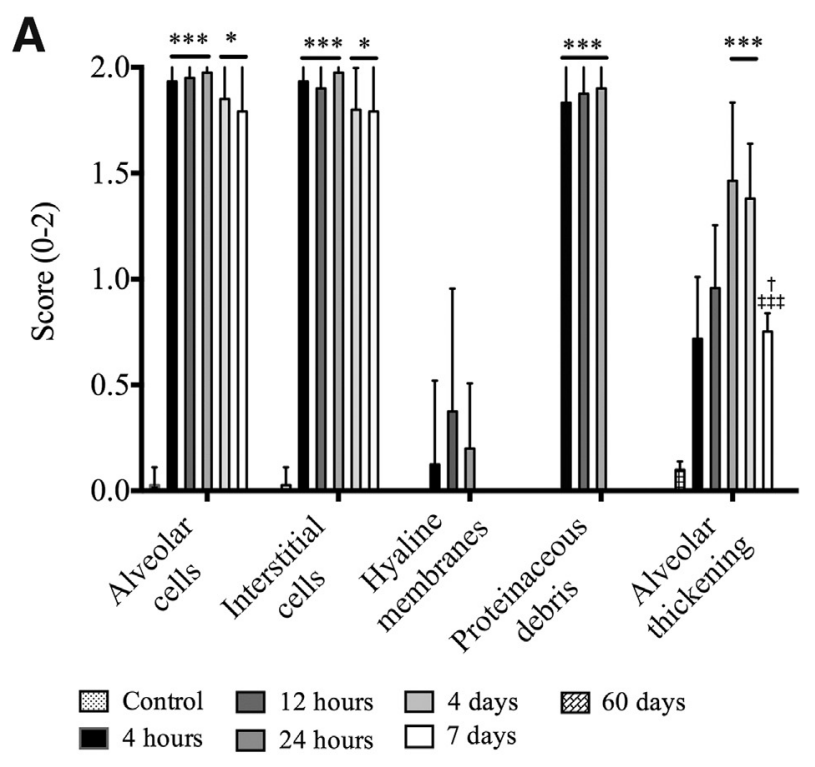

B

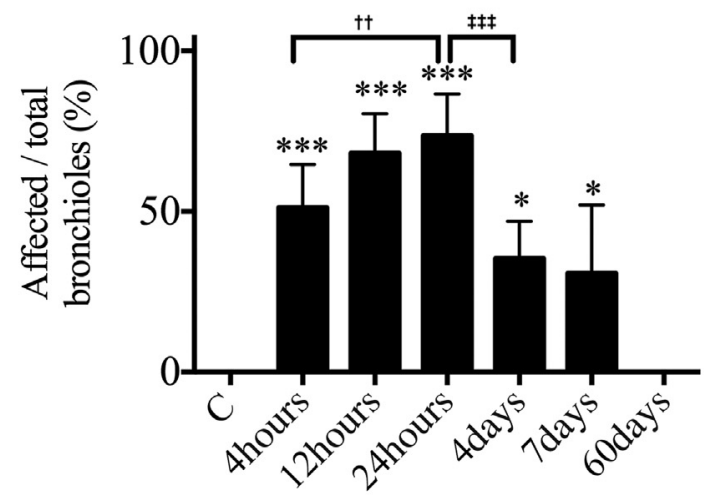

Time after instillation
Histological Scoring and Immunohistochemical Evaluation after Resolution of Initial ALI Markers

A semiquantitative score from 0 to 4 was developed ( 0 indicates no pathology; 1 , isolated; 2 , occasional; 3 , moderately intense; and 4, severe) to evaluate multinucleated giant cells, granulomas, and intra-alveolar buds of fibrosis (Masson bodies) in hematoxylin and eosinstained sections.

For collagen abundance, Masson's trichrome stain was used.

Abundance of myofibroblasts was evaluated using immunohistochemistry for $\alpha$-smooth muscle actin ( $\alpha$-SMA) by the immunoperoxidase technique. ${ }^{24}$ Endogenous peroxidase activity in lung sections was blocked with $3 \% \mathrm{H}_{2} \mathrm{O}_{2}$ for 10 minutes. Nonspecific reactivity was blocked with horse serum reactive for 10 minutes. Samples were then incubated with a mouse monoclonal anti- $\alpha$-SMA antibody (1:250) for 40 minutes at $37^{\circ} \mathrm{C}$. After repeated washing with phosphate-buffered saline, samples were incubated with rabbit biotinylated secondary antibody anti-mouse $\operatorname{IgG}$ for 25 minutes at $37^{\circ} \mathrm{C}$. This was followed by avidin-biotin amplification for 25 minutes and incubation with $3^{\prime}-3^{\prime}$
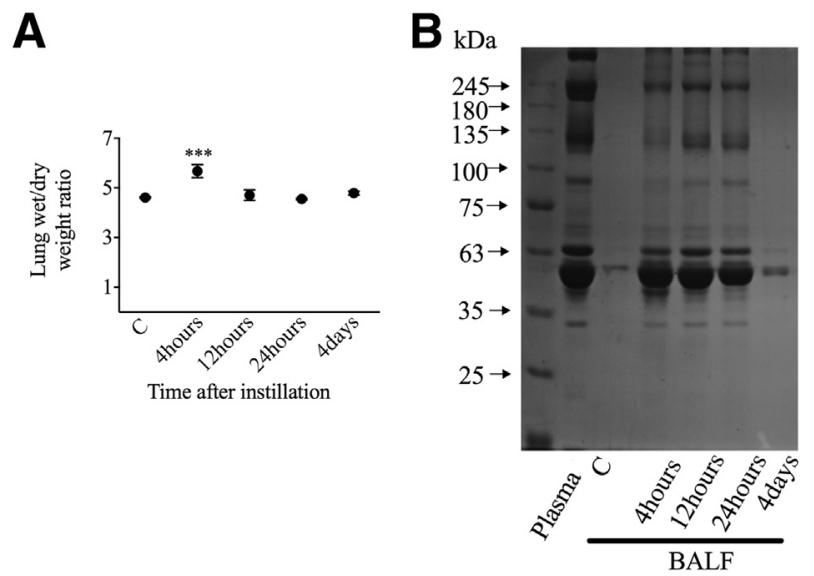

Figure 3 Scores for histological markers of acute lung injury (ALI). A: ALI score for each parameter shown in Table $1 .{ }^{21}$ The score for alveolar and interstitial cells is maximal at all times in the first week. A nonsignificant increase in the score for hyaline membranes is seen at 12 hours. The score for proteinaceous debris reaches its highest value at 4 hours, remains high at 24 hours, and returns to control values at 4 days. The score for alveolar thickening increases progressively, reaching a mean value of 1.5 at 24 hours and 4 days and decreasing at 7 days with a value similar to that at 4 hours. B: Percentage of affected bronchioles in the first week after instillation. At 4 hours, almost $50 \%$ of bronchioles are surrounded by inflammatory cell exudates. At 24 hours, this percentage further increases, with $70 \%$ of bronchioles being affected. At 4 and 7 days, the percentage of affected bronchioles decreases but remains higher than in controls. ${ }^{*} P<0.05,{ }^{* * *} P<0.001$ with respect to control; ${ }^{\dagger} P<0.01$ with respect to 4 days; ${ }^{\dagger \dagger} P<0.01$ with respect to 4 hours; ${ }^{\ddagger \ddagger} P<0.001$ with respect to 24 hours.

both lungs cannulated at the level of the trachea. The lungs were gradually inflated with air in 1-mL steps up to a pressure of $20 \mathrm{~cm} \mathrm{H}_{2} \mathrm{O}$ and then gradually deflated. These inflationdeflation cycles were recorded, and the $\Delta \mathrm{V} / \Delta \mathrm{P}$ ratio was obtained from the linear portion of the deflation curve.

Oxygen saturation was measured at the level of the tail of unanesthetized animals using a small animal oxymeter (Nonin Medical Inc., Plymouth, MN).
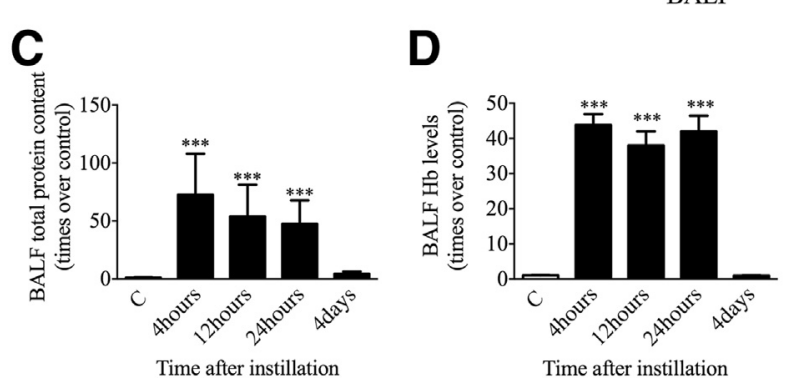

Figure 4 Markers of alveolar-capillary barrier derangement. A: Lung wet/ dry weight ratio is significantly increased at 4 hours and returns to control values at 12 hours. B: Representative Coomassie Blue-stained gel for a sample of plasma and bronchoalveolar lavage fluid (BALF) samples at baseline and at different times after instillation of whole gastric contents, showing that BALF total protein content is significantly increased at 4, 12, and 24 hours with a profile similar to that of plasma. At day 4 , the protein profile resembles the control $(C)$ profile. C: Quantitative changes in total protein concentration in BALF. A large increase in BALF total protein content is seen between 4 and 24 hours, with return to normal values at 4 days. D: Quantitative changes in BALF hemoglobin $(\mathrm{Hb})$ levels. A similar pattern to that of BALF total protein content is seen. Data are means \pm SD. ${ }^{* * * P}<0.001$. 


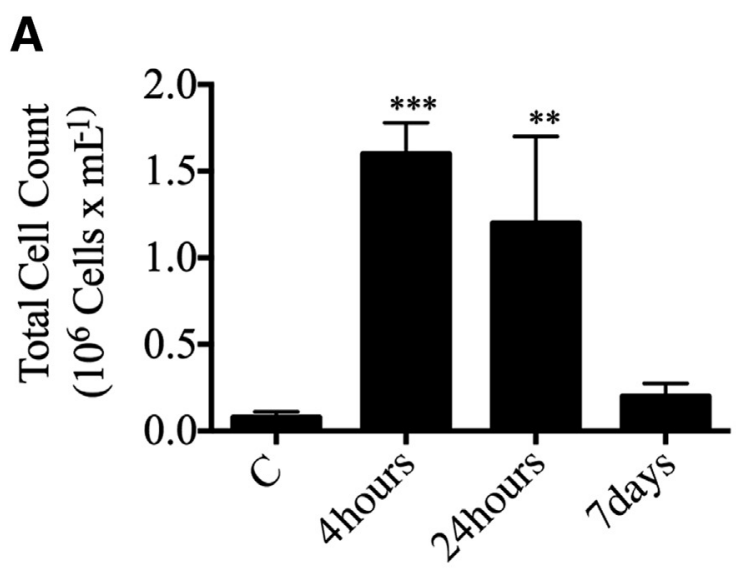

Time after Instillation

B

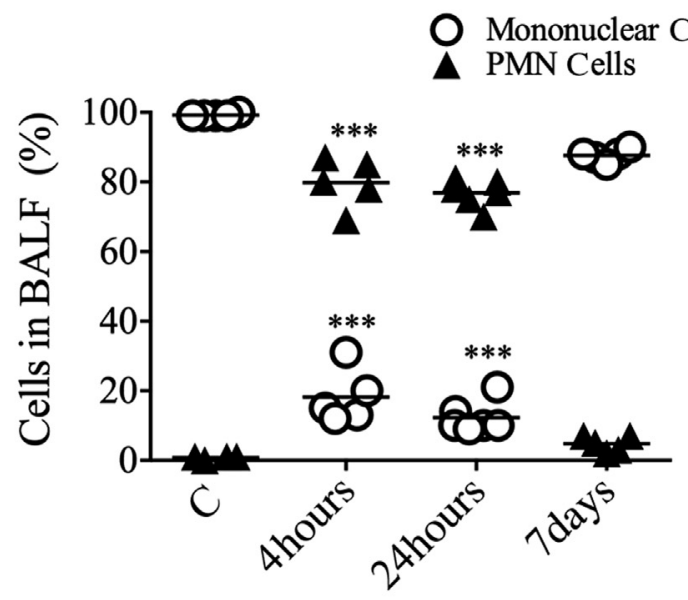

\section{Time after Instillation}

Figure 5 Total and differential cell count in bronchoalveolar lavage fluid (BALF). A: Significant increase in total cell count is seen at 4 and 24 hours with return to control values at day 7. B: Relative cell count in BALF shows a large increase in the percentage of polymorphonuclear (PMN) cells at 4 and 24 hours with a return to mononuclear cell predominance at day 7 . Data are means $\pm \mathrm{SD}(\mathbf{A})$ or means of individual values $(\mathbf{B}) .{ }^{* *} P<0.01$, $* * * P<0.001$

diaminobenzidine for 2 minutes at room temperature. $\mathrm{Nu}$ clear counterstain using Harris hematoxylin for 1 minute was followed by graded sequential dehydration in ethanol. Quantitative analysis of $\alpha$-SMA results: The area of positive $\alpha$-SMA stain in each Masson body was calculated and, for each animal, the average of 10 of these areas was reported. Positive stain in bronchial and vessel walls was considered the internal control.

\section{Statistical Analysis}

Normally distributed data were analyzed using one-way analysis of variance. The Kruskal-Wallis test was used for nonnormally distributed data. $P<0.05$ was considered significant. $^{25}$ Data were analyzed using GraphPad Prism 6 software (GraphPad Prism Inc., San Diego, CA).

\section{Results}

\section{Animal Model}

Preliminary studies showed the following: no significant difference in animal mortality when undiluted, 1:2, or 1:3 dilutions of gastric fluid were used (thus, undiluted fluid was used in this study); and no significant differences between right and left lung, nor between upper and lower lobes in injury distribution.

After careful titration of anesthesia, we achieved a mortality rate of $10 \%$. Mortality occurred immediately after instillation, mainly because of an anesthesia-induced decreased respiratory drive to cope with the volume of the aspirate. No significant changes in body weight were observed.

The percentage recovery of instilled saline during BAL was $67 \% \pm 7 \%$ (range, $64 \%$ to $69 \%$ ), with no difference between control and experimental groups.

\section{Lung Injury, Resolution, and Repair}

Acute Lung Injury

Histological evidence of tissue injury. Histological changes in the first 24 hours are shown in Figure 2. At 4
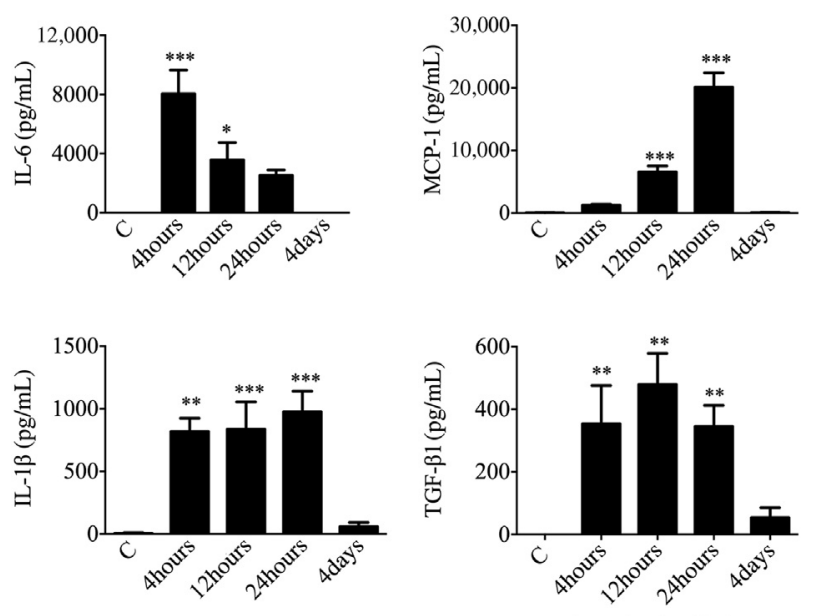

Time after instillation

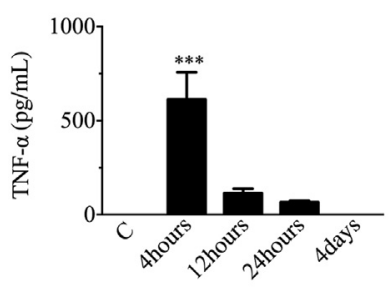

Time after instillation

Figure 6 Changes in cytokine and chemokine levels in bronchoalveolar lavage fluid (BALF). Levels of cytokines and chemokines in BALF show specific profiles. Proinflammatory cytokines IL- 6 and tumor necrosis factor (TNF)- $\alpha$ show a large and transient increase at 4 hours, decreasing rapidly to reach control $(C)$ values at 4 days, whereas IL- $1 \beta$ levels remain elevated until 24 hours to normalize only at 4 days. Levels of monocyte chemoattractant protein (MCP)1 increase progressively as IL- 6 and TNF- $\alpha$ levels decrease but also reach control levels at day 4 . The time course of the increase in the level of transforming growth factor (TGF)- $\beta 1$ is similar to that of IL- $1 \beta$. Data are means \pm SD. ${ }^{*} P<0.05,{ }^{* *} P<0.01$, and ${ }^{* * *} P<0.001$. 


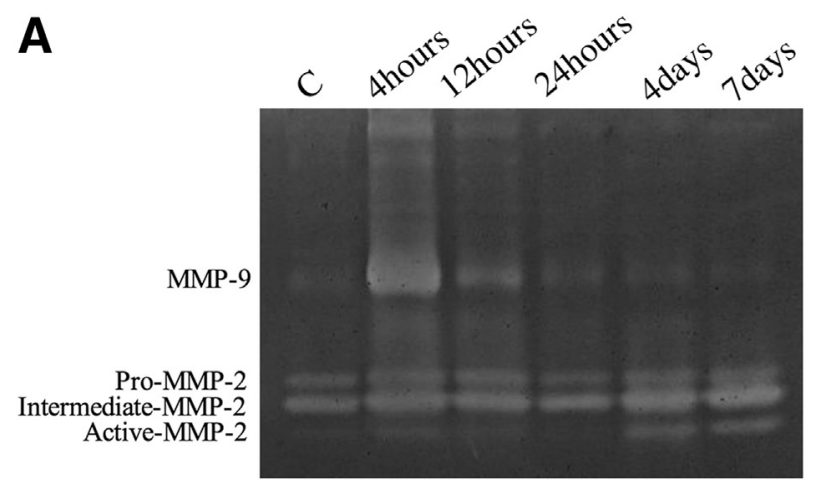

B

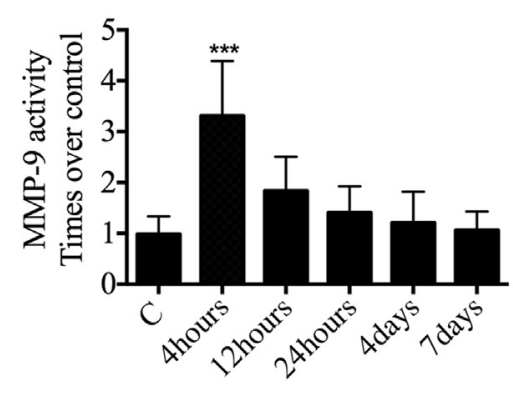

Time after instillation

\section{C}

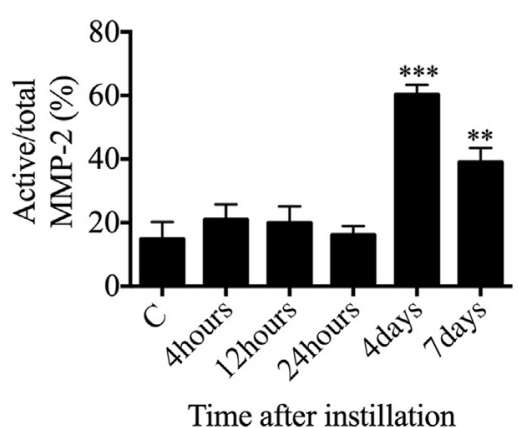

Figure 7 Changes in lung tissue matrix metalloproteinase (MMP)-9 and MMP-2 activities. A: Representative zymogram showing changes in lung tissue MMP-9 and MMP-2. MMP-9 activity is reported as times of increment of total gelatinolytic band density (approximately $90 \mathrm{kDa}$ ) over control (C). MMP-2 activity is reported as the ratio of active (approximately 68-kDa intermediate MMP-2 and approximately $62-\mathrm{kDa}$ full-active MMP-2) to inactive (approximately $72-k \mathrm{Da}$ pro-MMP-2) protein, in percentage. The densitometric analysis of six independent zymograms shows a peak of MMP-9 activity at 4 hours with a rapid decrease thereafter (B) and an increased MMP-2 activity at 4 and 7 days $(\mathbf{C})$. Data represent the means \pm SD (B and $\mathbf{C}) .{ }^{*} P<0.01$, $* * * P<0.001$.

hours, increased alveolar thickening by interstitial edema and inflammatory cell infiltration, along with abundant protein-rich intra-alveolar exudate containing neutrophils and red blood cells, was seen, adopting a peribronchiolar distribution. These changes became more intense at 12 and 24 hours, with patchy consolidation because of coalescence of affected areas. Microthrombi were frequently observed at these times. Hyaline membranes, the hallmark of human diffuse alveolar damage, were scarce, consistent with the knowledge that these structures are not usually seen during ALI in rodents. ${ }^{21}$

Figure $3 \mathrm{~A}$ shows the score for each of the ALI markers included in Table 1. Scores for alveolar and interstitial cells were maximal at all times during the first week. Hyaline membranes were scarce, with only a trend toward an increase at 12 hours. Proteinaceous debris was abundant, reaching maximal scores between 4 and 24 hours. Alveolar septa thickened progressively, approaching maximal score at 24 hours and 4 days, decreasing thereafter. Figure 3B shows that nearly $50 \%$ of bronchioles were affected at 4 hours, and in agreement with progression of damage, this percentage reached $70 \%$ at 24 hours, decreasing thereafter.

Markers of alveolar-capillary barrier derangement. The lung wet/dry weight ratio showed a large, but transient, increase at 4 hours (Figure 4A), whereas total protein concentration in BALF markedly increased between 4 and 24 hours (Figure 4C), in parallel with increased hemoglobin concentration (Figure 4D). A representative gel (Figure 4B) illustrated changes in the electrophoretic profile of plasma and BALF proteins after instillation. Control plasma and BALF samples differed in content and type of proteins, with few proteins visible in control BALF samples and a wide variety of proteins in plasma. At 4, 12, and 24 hours, there was a marked change in the protein profile of BALF, with addition of proteins of different molecular sizes, similar to those seen in plasma, evidencing a significant transendothelial passage of blood proteins. At day 4, largemolecular-weight proteins disappeared from BALF, which then exhibited a protein profile similar to that of controls.

Inflammatory response. Changes in total and differential cell count in BALF were analyzed (Figure 5). A 15- to 20-fold increase in total cell count was seen in the first 24 hours (Figure 5A), with polymorphonuclear cell predominance (Figure 5B). By day 7, there was a return to mononuclear cell predominance. The changes in BALF cytokine levels were transient (Figure 6). At 4 hours, levels of IL-6 and tumor necrosis factor- $\alpha$ were markedly increased but showed a rapid decline at 12 hours. IL-1 $\beta$ levels were also elevated at 4 hours but remained high until 24 hours. Monocyte chemoattractant protein-1 levels increased slowly, reaching a peak at 24 hours, with a similar profile to that of IL-1 $\beta$. Concentrations of all measured cytokines were normal at day 4 .

Changes in activity of MMP-9 and MMP-2 were analyzed. Zymograms (Figure 7A) showed a transient increase in the size of the 90-kDa gelatinolytic band (total MMP-9) at 4 hours

Table 2 Correlations between MMP-9 Activity and Inflammatory Markers

\begin{tabular}{lll}
\hline Marker & $r$ & $P$ value \\
\hline Neutrophils & 0.819 & 0.08 \\
IL-6 & 0.967 & 0.007 \\
TNF- $\alpha$ & 0.988 & 0.001 \\
IL-1 $\beta$ & 0.616 & 0.355 \\
\hline
\end{tabular}

MMP, matrix metalloproteinase; TNF, tumor necrosis factor. 
Table 3 EELV, Lung Compliance, and Arterial 0xygen Saturation at 12 to 24 Hours and at 7 Days after Instillation

\begin{tabular}{lccc}
\hline Parameter & Control $(n=6)$ & $12-24$ Hours $(n=5)$ & 7 Days $(n=6)$ \\
\hline EELV $(\mathrm{mL})$ & $2.8 \pm 0.96$ & $1.33 \pm 0.8^{*}$ & $3.9 \pm 1$ \\
$\Delta \mathrm{V} / \Delta \mathrm{P}$ ratio $\left(\mathrm{mL} / \mathrm{cm} \mathrm{H} \mathrm{H}_{2} \mathrm{O}\right)$ & $0.85 \pm 0.14$ & $0.61 \pm 0.14^{*}$ & $0.90 \pm 0.21$ \\
Oxygen saturation $(\%)$ & $95.6 \pm 1.7$ & $81 \pm 7^{*}$ & $\mathrm{ND}$ \\
\hline
\end{tabular}

Data are given as means \pm SD.

${ }^{*} P<0.05$ vs control values.

EELV, end expiratory lung volume; ND, not determined.

and a new gelatinolytic band of $62 \mathrm{kDa}$ (active fragment of MMP-2) at days 4 and $7 .^{26}$ Densitometric analysis showed a timely dissociated increase in activity of these MMPs, with an increase in MMP-9 total activity at 4 hours that rapidly returned to control levels (Figure $7 \mathrm{~B}$ ), and an increase in MMP-2 activity only at days 4 and 7 (Figure 7C). MMP-9 activity correlated with polymorphonuclear neutrophil cells and some proinflammatory cytokines in BALF (Table 2).

Evidence of physiological dysfunction. Table 3 shows a significant reduction in arterial oxygen saturation, end expiratory lung volume, and lung compliance at 12 to 24 hours after instillation in comparison with controls. Table 3 also shows that end expiratory lung volume and lung compliance are back to control values at day 7 .

\section{Resolution and repair}

\section{Histological scoring and immunohistochemical evaluation} after resolution of initial ALI markers. Histological changes at days 4 and 7 after instillation were studied. Foreignbody giant cells, either isolated (Figure 8B) or forming granulomas (Figure 8E), were frequently observed. Intraalveolar buds of granulation tissue, characteristic of $\mathrm{OP},{ }^{27,28}$ were abundant (Figure 8, C-E). These Masson bodies sometimes contained granulomas and giant cells (Figure 8, C and F). Control lung is shown in Figure 8A. Markers of ALI, as described in the first 24 hours, were no longer observed at days 4 and 7.

$\alpha$-SMA-positive structures were observed (Figure 9). Although control samples exhibited staining localized to the wall of bronchioles and blood vessels only (Figure 9, A and D), 4- and 7-day samples (Figure 9, B and C) showed brownstained myofibroblasts intermixed with collagen fibers inside Masson bodies (Figure 9E). Granuloma-containing Masson bodies were frequently observed (Figure 9F). The mean area of $\alpha$-SMA-positive stain in Masson bodies was $61.3 \% \pm 15.9 \%$ at day 4 and $52.3 \% \pm 14 \%$ at day $7(P<0.05)$.

Collagen staining showed abundant collagen fibers inside Masson bodies intermixed with myofibroblasts at both 4 and 7 days (Figure 10, B-D). Some of the Masson bodies were obliterating bronchioles (Figure 10B). Control showing positive staining in bronchiolar wall are shown in Figure 10A. Results of semiquantitative evaluation of giant cells, granulomas, Masson bodies, and collagen are shown in Figure 11.

At day 60, normal lung architecture was found, with disappearance of inflammatory elements, food particles, granulomas, and Masson bodies (Figure 12).

\section{Discussion}

We successfully developed a reproducible animal model of gastric aspiration with low mortality. A single dose of whole gastric content in the rat lung results in a sequence of alterations, starting with severe derangement of the alveolarcapillary barrier and an intense inflammatory and hemorrhagic infiltration that thickens the interstitium and flows into the alveolar spaces, with significant impairment of physiological parameters.

As initial markers of ALI resolve, an organization process ensues involving most of the constitutive elements of $\mathrm{OP}$ (namely, intraluminal plugs of myofibroblasts and collagen

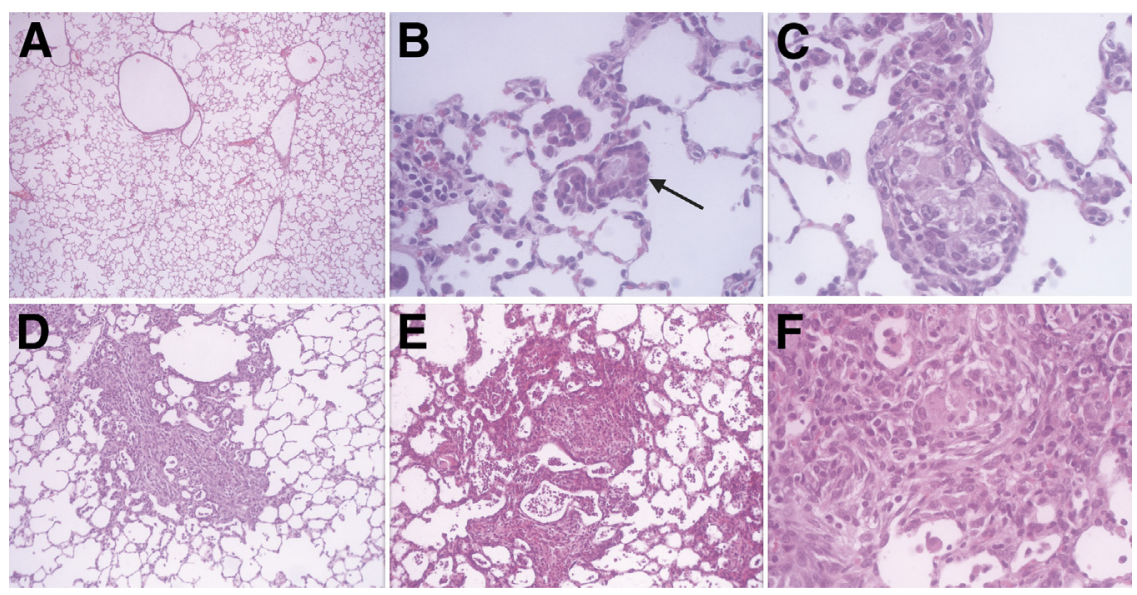

Figure 8 Light microscopy (hematoxylin and eosin stain) of control lung and 4 and 7 days after whole gastric content instillation. Control (A), foreign-body giant cells (arrow) at 7 days (B), Masson body containing granuloma and giant cells at 7 days (C), intra-alveolar buds of granulation tissue (Masson body), corresponding to organizing pneumonia ( $\mathbf{D}$ and $\mathbf{E})$, and a high-power view of a Masson body containing giant cells at day 4 (F). Original magnification: $\times 40($ A, B, and D); $\times 200$ $(C) ; \times 100(\mathbf{E}) ; \times 400(\mathbf{F})$. 

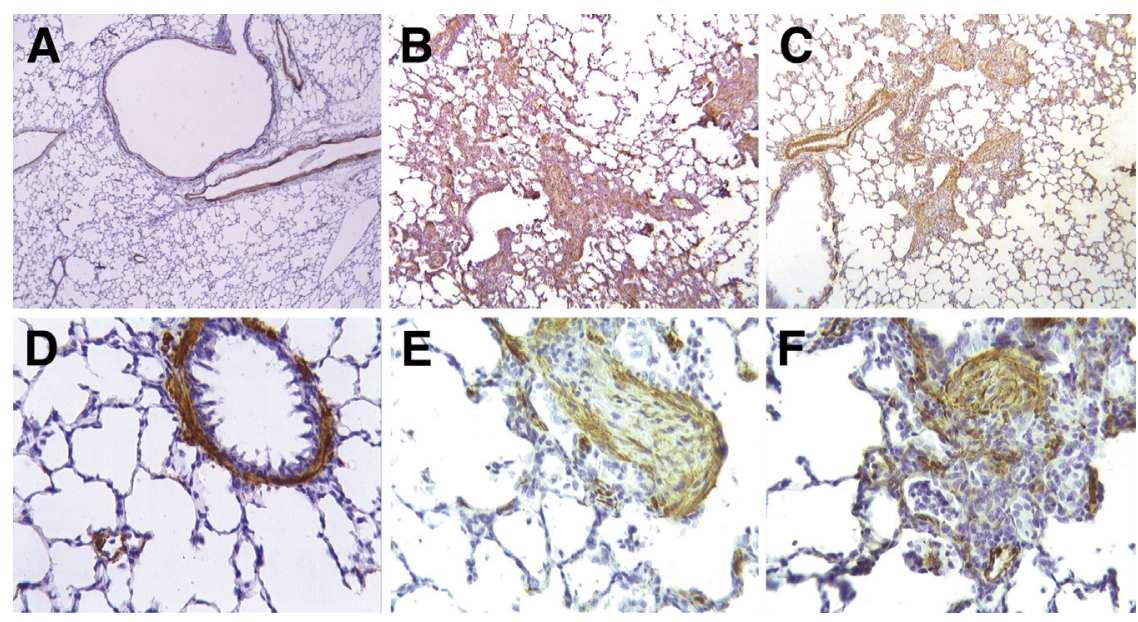

Figure 9 Immunohistochemical staining of myofibroblasts ( $\alpha$-smooth muscle actin). A and $\mathbf{D}$ : Control samples showing positive staining in bronchioles and vessels. The 4-day (B) and 7-day (C) samples showing abundant brown-stained myofibroblasts organized in buds of granulation tissue. E: High-power view of a Masson body showing the concentric orientation of myofibroblasts inside the intra-alveolar buds. F: Masson body containing granuloma. Original magnification: $\times 40(\mathbf{A}-\mathbf{C}) ; \times 400(\mathbf{D}-\mathbf{F})$. fibers affecting small bronchioles, alveolar ducts, and peribronchiolar alveolar spaces). ${ }^{27,28}$ Foreign-body giant cells isolated or forming granulomas are also seen. Thus, in our model, the OP pattern represents organization of the initial inflammatory reaction, whereas in humans, diffuse alveolar damage and OP are frequently diagnosed as independent patterns in association with aspiration. ${ }^{29}$ Furthermore, our OP pattern is self-limited because, at day 60 after instillation, lung architecture was restored. This continuum of alterations shares significant similarities with the phases described in normal wound healing. ${ }^{28,30}$

Previous studies on the consequences of aspiration are limited in their ability to model human aspiration. To reduce mortality, investigators have used individual components of gastric fluid, ${ }^{6-8,10,11}$ localized instillation of small doses of acid or gastric fluid, ${ }^{14,15}$ or supportive mechanical ventilation. ${ }^{7}$ By adequate titration of anesthesia and careful management of the airway after orotracheal instillation of whole gastric contents, we developed a reproducible, low-mortality model of aspiration that does not require mechanical ventilation, thus avoiding the possibility of adding a second injury hit. We found that a delicate balance exists between the level of anesthesia that allows for intubation and instillation and the maintenance of a sufficient respiratory drive to cope with the instilled volume, because the few observed deaths occurred immediately after instillation.

A recent publication has addressed the problem of modeling human ALI in animals ${ }^{21}$ and has recommended that at least three of four main features should be present to determine that ALI has occurred (namely, histological evidence of tissue injury, derangement of the alveolar capillary barrier, an inflammatory response, and evidence of physiological dysfunction). Our model meets all four main features for experimental ALI. By using several methodological approaches to support each of these main features, we provide strong evidence of the presence of ALI in a model that is highly relevant in the clinical setting, because aspiration of gastric contents is a well-recognized risk factor for the development of ALI/acute respiratory distress syndrome in humans.
Interestingly, we found similarities with the pattern of resolution of ALI reported in models using acid alone. ${ }^{1,7}$ Lung water content was restored rapidly, whereas concentration of high-molecular-weight proteins in the alveolar spaces remained elevated for a longer time, reflecting known differences in protein and water clearance in ALI. ${ }^{31}$ As in most ALI models, the initial inflammatory cell infiltration was predominantly neutrophilic and was accompanied by an early and transient increase in the level of proinflammatory cytokines. The time course of changes in monocyte chemoattractant protein-1 levels, increasing later than proinflammatory cytokines but preceding the return to mononuclear cell predominance and granuloma formation, supports a role of this cytokine in monocyte recruitment $^{32}$ and granuloma formation. ${ }^{11}$

Because most animals survived the initial stage of ALI, we were able to appreciate an ensuing organization and repair process similar to wound healing. ${ }^{30,33,34}$ A large number of

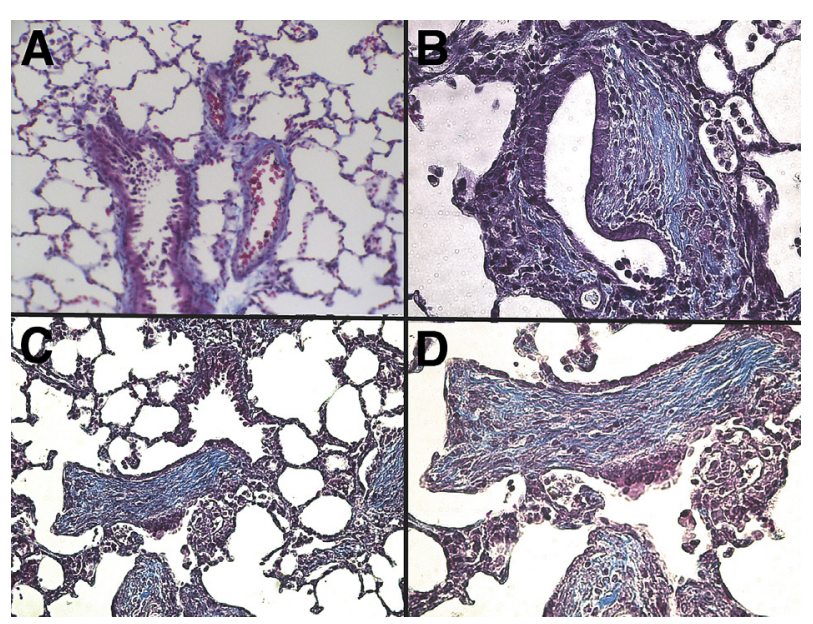

Figure 10 Masson's trichrome staining in lung tissue samples 4 to 7 days after a single dose of gastric contents. A: Control showing positive staining in bronchiolar walls. B: Blue-stained Masson body obliterating a bronchiole. $\mathbf{C}$ and $\mathbf{D}$ : Intra-alveolar Masson bodies with collagen fibers on the inside at different magnifications. Original magnification: $\times 40($ A); $\times 400$ (B and D); $\times 100($ C). 


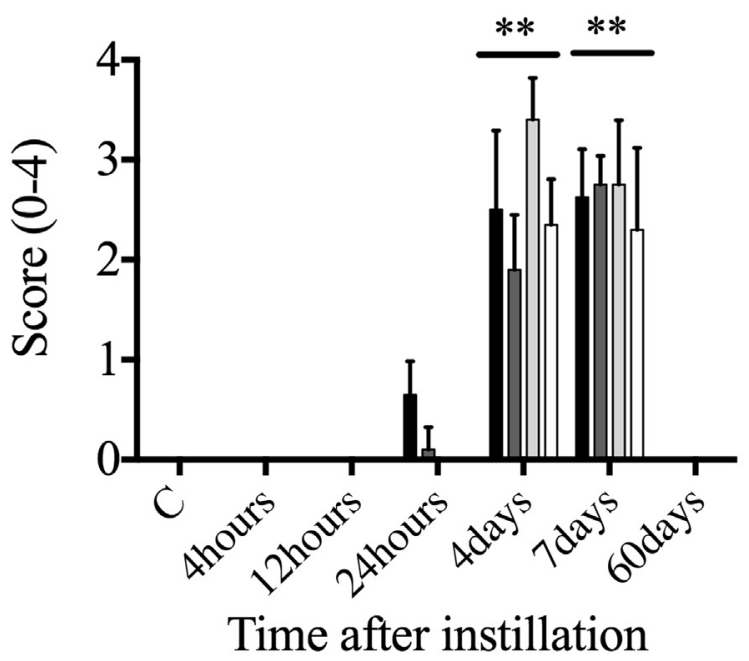

\section{- Granulomas \\ Masson bodies \\ Foreign-body giant cells \\ Collagen staining}

Figure 11 Score for Masson bodies, foreign-body giant cells, granulomas, and abundance of collagen staining. Masson bodies, foreign-body giant cells, and granulomas are present at 4 and 7 days only. Collagen staining score is also increased at 4 and 7 days. ${ }^{*} P<0.01$.

myofibroblasts inside Masson bodies were seen at days 4 and 7, a time frame similar to that required for granulation tissue formation during wound repair. ${ }^{35}$ Myofibroblasts organized themselves in concentric rings, generating well-defined, collagen-containing, intra-alveolar buds of granulation tissue, the histological hallmark of OP. ${ }^{28}$ The presence of these cells was preceded by an increase in the concentration of transforming growth factor- $\beta 1$, a key cytokine in myofibroblast activation. $^{36-38}$

The relationship between gastric content aspiration and OP in humans is not well defined. ${ }^{18,39}$ In a series of surgical lung specimens with evidence of gastric content aspiration, Mukhopadhyay and Katzenstein ${ }^{18}$ showed OP in nearly $90 \%$ of specimens. Whether $\mathrm{OP}$ in those cases was a de novo process or, instead, represented organization of a previous exudative reaction is unknown, because of the one-time snapshot character of human lung biopsy specimens. ${ }^{33}$ Our study of the time course of the effects of gastric content instillation, starting at early stages of injury, was of paramount importance to convincingly show that OP arises as a way of organization of the early intense inflammatory exudate that characterizes aspiration-induced ALI in the rat. Our results further help in understanding the results of Mukhopadhyay and Katzenstein, ${ }^{18}$ by contextualizing the presence of OP after aspiration as part of the resolution and repair process in aspiration-induced ALI.

It is recognized that a proportion of patients with aspirationinduced ALI can develop pathological fibrosis. ${ }^{1}$ In addition, studies in mice instilled with hydrochloric acid have shown some degree of fibrosis when studied in the first 2 weeks. ${ }^{4,7}$ In our study, however, we observed resolution of ALI markers, followed by the development of an OP pattern, with reversible fibrosis and restoration of normal lung architecture when enough time elapsed after instillation. Thus, we speculate that the fibrosis described in short-term studies in mice after hydrochloric acid instillation ${ }^{4,7}$ could also be reversible if animals would have been studied at a later time. It is likely that in humans who do develop fibrosis after a single event of aspiration, silent repeated aspiration or contributing factors, such as comorbidities or therapies (ie, drugs, oxygen, or mechanical ventilation), may play a role in determining whether fibrosis will develop in subjects with a genetic predisposition. In addition, recent evidence shows that increased age is associated with an impaired ability to reverse bleomycin-induced fibrosis. The fact that, in our study, young adult animals were used could have played a role in fibrosis reversibility. ${ }^{40}$

Among several possible factors involved in limiting fibrosis development in our study, early restoration of transforming growth factor- $\beta 1$ levels may have a role, because its elevated levels contribute to persistent fibrosis by inhibition of myofibroblast apoptosis. ${ }^{41}$ Although our study was not designed to evaluate the fate of myofibroblasts, the results obtained with the $\alpha$-SMA stain support an early reduction in myofibroblasts per Masson body when comparing 4- with 7-day samples. Increased collagen turnover by MMPs is also known to play a role in fibrosis modulation. ${ }^{42}$ Changes in MMP activity, reported in some animal models of $\mathrm{ALI},{ }^{43}$ have not been studied after gastric fluid instillation. In our study, although with a different time course, MMP-2 and MMP-9 showed increased activity. Although MMP-9 activity increased during the acute phase of injury, MMP-2 activity increased later, when OP was present. This finding supports the idea that these MMPs may be active at different times after injury, with MMP-9 linked to the initial inflammatory reaction and MMP-2 to the repair process, an interpretation supported by similar results in rabbits with bleomycin-induced pulmonary fibrosis. ${ }^{44,45}$

The role of MMP-9 in ALI remains controversial. Although in one study, its inhibition attenuated ventilator-induced lung

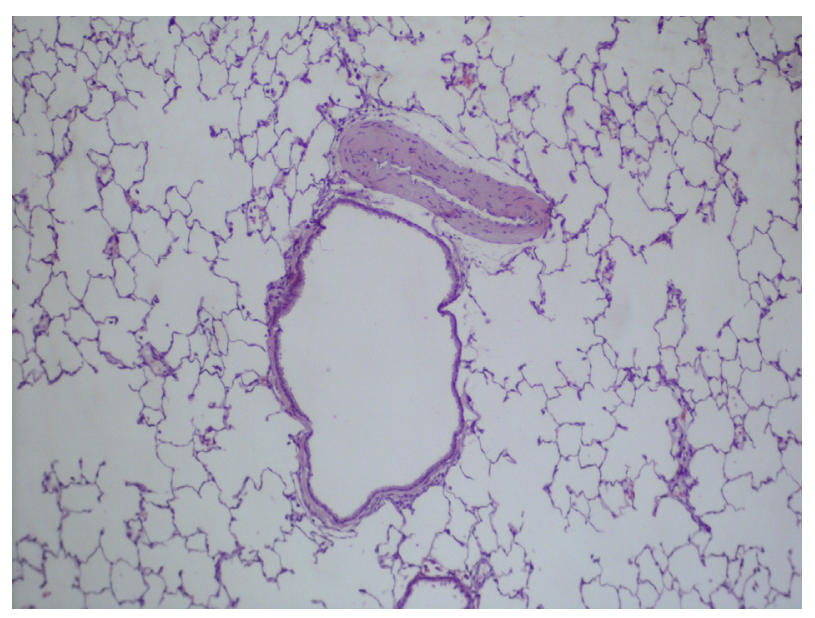

Figure 12 Light microscopy (hematoxylin and eosin stain of rat lung at day 60 after whole gastric content instillation shows normal lung architecture. Original magnification, $\times 40$. 
injury, ${ }^{46}$ in an ozone-induced airway inflammation model, the deletion of its encoding gene led to increased levels of inflammatory markers. ${ }^{47}$ Neutrophils are the main source of MMP-9, releasing it on stimulation. ${ }^{48}$ In our study, the early increase in MMP-9 activity correlated with levels of polymorphonuclear cells, IL-6, and tumor necrosis factor- $\alpha$. The time course of changes in MMP-9 activity paralleled the time course of changes in markers of alveolar barrier derangement. It is likely that MMP-9 plays a role in the derangement of the alveolar capillary barrier, because type IV collagen, an important component of the basement membrane, is one of its main substrates. ${ }^{49}$ On the basis of in vitro studies, this effect could be beneficial because it contributes to migration of repairing cells. ${ }^{50}$

MMP-2 activity did not increase during the initial stage of ALI in our model. Instead, it increased only when OP was present. Interestingly, it has been shown that lung biopsy specimens from patients with OP also exhibit increased MMP-2 activity. ${ }^{45,49}$ Given the reversible nature of fibrosis in OP and the fact that the active form of MMP-2 is markedly increased during the alveolar stage of fetal lung development, it has been proposed that MMP-2 is necessary for alveolar reconstruction. ${ }^{49}$ Accordingly, we propose that an increased activity of MMP-2 may also contribute to fibrosis limitation after a single instillation of gastric contents. Future studies examining natural inhibitors of MMPs in the setting of gastric aspiration are warranted.

Another relevant observation in our study is the presence of multinucleated giant cells, both isolated and forming granulomas after the first 24 hours, in agreement with studies using gastric food particles in animals ${ }^{11,16}$ and in human cases of aspiration. ${ }^{18,51}$ In our study, most food particles were observed free in the alveolar spaces during the initial stage of ALI and inside multinucleated giant cells at days 4 and 7 . Interestingly, at day 60 , no significant particulate material was found, suggesting that macrophages, giant cells, and granulomas are important for compartmentalization and elimination of the foreign material. Some authors have suggested that to avoid chronic fibrosis, the lung must be able to eliminate the injurious agent and restore both endothelial and epithelial cells. ${ }^{33}$ In our model, the lung's ability to eliminate instilled food particles could have also contributed to limit fibrosis. It is possible, however, that repeated episodes of aspiration might not have this result.

In summary, we developed an animal model to study the complex pathogenesis of gastric content aspiration. We have demonstrated that, in the rat, the consequences of aspiration are manifested as a continuum of alterations that act in an orchestrated and somewhat overlapping manner, starting with severe ALI and evolving toward an OP pattern that is reversible, leaving the lung with normal architecture. In addition to identifying markers of staging and severity, this model reveals that OP is part of the repair process in the setting of aspiration-induced injury. Given the high prevalence of aspiration in the clinical setting, this model may be useful to better understand the pathogenesis of aspiration in humans and to evaluate new therapeutic interventions.

\section{References}

1. Raghavendran K, Nemzek J, Napolitano LM, Knight PR: Aspirationinduced lung injury. Crit Care Med 2011, 39:818-826

2. Metheny NA, Clouse RE, Chang YH, Stewart BJ, Oliver DA, Kollef MH: Tracheobronchial aspiration of gastric contents in critically ill tube-fed patients: frequency, outcomes, and risk factors. Crit Care Med 2006, 34:1007-1015

3. Marik PE: Aspiration pneumonitis and aspiration pneumonia. N Engl J Med 2001, 344:665-671

4. Amigoni M, Bellani G, Scanziani M, Masson S, Bertoli E, Radaelli E, Patroniti N, Di Lelio A, Pesenti A, Latini R: Lung injury and recovery in a murine model of unilateral acid aspiration: functional, biochemical, and morphologic characterization. Anesthesiology 2008 , 108:1037-1046

5. Kennedy TP, Johnson KJ, Kunkel RG, Ward PA, Knight PR, Finch JS: Acute acid aspiration lung injury in the rat: biphasic pathogenesis. Anesth Analg 1989, 69:87-92

6. Knight PR, Druskovich G, Tait AR, Johnson KJ: The role of neutrophils, oxidants, and proteases in the pathogenesis of acid pulmonary injury. Anesthesiology 1992, 77:772-778

7. Patel BV, Wilson MR, Takata M: Resolution of acute lung injury and inflammation: a translational mouse model. Eur Respir J 2012, 39 : 1162-1170

8. Knight PR, Rutter T, Tait AR, Coleman E, Johnson K: Pathogenesis of gastric particulate lung injury: a comparison and interaction with acidic pneumonitis. Anesth Analg 1993, 77:754-760

9. Teabeaut JR 2nd: Aspiration of gastric contents: an experimental study. Am J Pathol 1952, 28:51-67

10. Knight PR, Davidson BA, Nader ND, Helinski JD, Marschke CJ, Russo TA, Hutson AD, Notter RH, Holm BA: Progressive, severe lung injury secondary to the interaction of insults in gastric aspiration. Exp Lung Res 2004, 30:535-557

11. Raghavendran K, Davidson BA, Mullan BA, Hutson AD, Russo TA, Manderscheid PA, Woytash JA, Holm BA, Notter RH, Knight PR: Acid and particulate-induced aspiration lung injury in mice: importance of MCP-1. Am J Physiol Lung Cell Mol Physiol 2005, 289:L134-L143

12. Mendelson CL: The aspiration of stomach contents into the lungs during obstetric anesthesia. Am J Obstet Gynecol 1946, 52:191-205

13. Davidson BA, Knight PR, Wang Z, Chess PR, Holm BA, Russo TA, Hutson A, Notter RH: Surfactant alterations in acute inflammatory lung injury from aspiration of acid and gastric particulates. Am J Physiol Lung Cell Mol Physiol 2005, 288:L699-L708

14. Appel JZ 3rd, Lee SM, Hartwig MG, Li B, Hsieh CC, Cantu E 3rd, Yoon Y, Lin SS, Parker W, Davis RD: Characterization of the innate immune response to chronic aspiration in a novel rodent model. Respir Res 2007, 8:87

15. Downing TE, Sporn TA, Bollinger RR, Davis RD, Parker W, Lin SS: Pulmonary histopathology in an experimental model of chronic aspiration is independent of acidity. Exp Biol Med (Maywood) 2008 , 233:1202-1212

16. Hartwig MG, Appel JZ, Li B, Hsieh CC, Yoon YH, Lin SS, Irish W, Parker W, Davis RD: Chronic aspiration of gastric fluid accelerates pulmonary allograft dysfunction in a rat model of lung transplantation. J Thorac Cardiovasc Surg 2006, 131:209-217

17. Katzenstein A-L: Acute lung injury patterns: diffuse alveolar damage and bronchiolitis obliterans-organizing pneumonia. Edited by Katzenstein A-L. Katzenstein and Askin's Surgical Pathology of Non-neoplastic Lung Disease. 3rd Edition. Philadelphia, PA, Saunders, 1997, pp. 14-47

18. Mukhopadhyay S, Katzenstein AL: Pulmonary disease due to aspiration of food and other particulate matter: a clinicopathologic study 
of 59 cases diagnosed on biopsy or resection specimens. Am J Surg Pathol 2007, 31:752-759

19. Travis WD: Non-Neoplastic Disorders of the Lower Respiratory Tract. Edited by Travis WD, Colby TV, Koss MN, Rosado-de Christenson ML, Muller NL, King TE. American Registry of Pathology, 2002

20. Lee JS, Collard HR, Raghu G, Sweet MP, Hays SR, Campos GM, Golden JA, King TE Jr: Does chronic microaspiration cause idiopathic pulmonary fibrosis? Am J Med 2010, 123:304-311

21. Matute-Bello G, Downey G, Moore BB, Groshong SD, Matthay MA, Slutsky AS, Kuebler WM: An official American Thoracic Society workshop report: features and measurements of experimental acute lung injury in animals. Am J Respir Cell Mol Biol 2011, 44:725-738

22. Bradford MM: A rapid and sensitive method for the quantitation of microgram quantities of protein utilizing the principle of protein-dye binding. Anal Biochem 1976, 72:248-254

23. Toth M, Sohail A, Fridman R: Assessment of gelatinases (MMP-2 and MMP-9) by gelatin zymography. Methods Mol Biol 2012, 878:121-135

24. Zhang K, Rekhter MD, Gordon D, Phan SH: Myofibroblasts and their role in lung collagen gene expression during pulmonary fibrosis: a combined immunohistochemical and in situ hybridization study. Am J Pathol 1994, 145:114-125

25. Sokal RR, Rohlf FJ: Biometry, the Principles and Practice of Statistics in Biological Research. San Francisco, Freeman, 1981

26. Stawowy P, Margeta C, Kallisch H, Seidah NG, Chretien M, Fleck E, Graf K: Regulation of matrix metalloproteinase MT1-MMP/MMP-2 in cardiac fibroblasts by TGF-beta1 involves furin-convertase. Cardiovasc Res 2004, 63:87-97

27. Epler GR, Colby TV, McLoud TC, Carrington CB, Gaensler EA: Bronchiolitis obliterans organizing pneumonia. N Engl J Med 1985, 312:152-158

28. Cordier JF: Cryptogenic organising pneumonia. Eur Respir J 2006, $28: 422-446$

29. Yousem SA, Faber C: Histopathology of aspiration pneumonia not associated with food or other particulate matter: a clinicopathologic study of 10 cases diagnosed on biopsy. Am J Surg Pathol 2011, 35:426-431

30. Demidova-Rice TN, Hamblin MR, Herman IM: Acute and impaired wound healing: pathophysiology and current methods for drug delivery, part 2: role of growth factors in normal and pathological wound healing: therapeutic potential and methods of delivery. Adv Skin Wound Care 2012, 25:349-370

31. Folkesson HG, Nitenberg G, Oliver BL, Jayr C, Albertine KH, Matthay MA: Upregulation of alveolar epithelial fluid transport after subacute lung injury in rats from bleomycin. Am J Physiol 1998, 275: L478-L490

32. Deshmane SL, Kremlev S, Amini S, Sawaya BE: Monocyte chemoattractant protein-1 (MCP-1): an overview. J Interferon Cytokine Res 2009, 29:313-326

33. Strieter RM: What differentiates normal lung repair and fibrosis? Inflammation, resolution of repair, and fibrosis. Proc Am Thorac Soc 2008, 5:305-310

34. Clark RA: Basics of cutaneous wound repair. J Dermatol Surg Oncol 1993, 19:693-706

35. Singer AJ, Clark RA: Cutaneous wound healing. N Engl J Med 1999, 341:738-746
36. Hinz B, Phan SH, Thannickal VJ, Galli A, Bochaton-Piallat ML, Gabbiani G: The myofibroblast: one function, multiple origins. Am J Pathol 2007, 170:1807-1816

37. Phan SH: Genesis of the myofibroblast in lung injury and fibrosis. Proc Am Thorac Soc 2012, 9:148-152

38. Garcia de Alba C, Buendia-Roldan I, Salgado A, Becerril C, Ramirez R, Gonzalez Y, Checa M, Navarro C, Ruiz V, Pardo A, Selman M: Fibrocytes contribute to inflammation and fibrosis in chronic hypersensitivity pneumonitis through paracrine effects. Am J Respir Crit Care Med 2015, 191:427-436

39. Morehead RS: Gastro-oesophageal reflux disease and non-asthma lung disease. Eur Respir Rev 2009, 18:233-243

40. Hecker L, Logsdon NJ, Kurundkar D, Kurundkar A, Bernard K, Hock T, Meldrum E, Sanders YY, Thannickal VJ: Reversal of persistent fibrosis in aging by targeting Nox4-Nrf2 redox imbalance. Sci Transl Med 2014, 6:231ra47

41. Phan SH: The myofibroblast in pulmonary fibrosis. Chest 2002, 122: 286S-289S

42. Pardo A, Selman M: Role of matrix metalloproteinases in pulmonary fibrosis. Matrix Metalloproteinases in Tissue Remodeling and Inflammation. Edited by Lagente V, Boichot E. Basel, Switzerland, Springer Birkhauser, 2008, pp. 39-55

43. Corbel M, Boichot E, Lagente V: Role of gelatinases MMP-2 and MMP-9 in tissue remodeling following acute lung injury. Braz J Med Biol Res 2000, 33:749-754

44. Yaguchi T, Fukuda Y, Ishizaki M, Yamanaka N: Immunohistochemical and gelatin zymography studies for matrix metalloproteinases in bleomycin-induced pulmonary fibrosis. Pathol Int 1998, 48:954-963

45. Suga M, Iyonaga $\mathrm{K}$, Okamoto $\mathrm{T}$, Gushima $\mathrm{Y}$, Miyakawa $\mathrm{H}$, Akaike T, Ando M: Characteristic elevation of matrix metalloproteinase activity in idiopathic interstitial pneumonias. Am J Respir Crit Care Med 2000, 162:1949-1956

46. Foda HD, Rollo EE, Drews M, Conner C, Appelt K, Shalinsky DR, Zucker S: Ventilator-induced lung injury upregulates and activates gelatinases and EMMPRIN: attenuation by the synthetic matrix metalloproteinase inhibitor, Prinomastat (AG3340). Am J Respir Cell Mol Biol 2001, 25:717-724

47. Yoon HK, Cho HY, Kleeberger SR: Protective role of matrix metalloproteinase-9 in ozone-induced airway inflammation. Environ Health Perspect 2007, 115:1557-1563

48. Grommes J, Soehnlein O: Contribution of neutrophils to acute lung injury. Mol Med 2011, 17:293-307

49. Fukuda Y, Ishizaki M, Kudoh S, Kitaichi M, Yamanaka N: Localization of matrix metalloproteinases-1, -2 , and -9 and tissue inhibitor of metalloproteinase-2 in interstitial lung diseases. Lab Invest 1998, 78:687-698

50. Legrand C, Gilles C, Zahm JM, Polette M, Buisson AC, Kaplan H, Birembaut P, Tournier JM: Airway epithelial cell migration dynamics: MMP-9 role in cell-extracellular matrix remodeling. J Cell Biol 1999, 146:517-529

51. Barnes TW, Vassallo R, Tazelaar HD, Hartman TE, Ryu JH: Diffuse bronchiolar disease due to chronic occult aspiration. Mayo Clin Proc 2006, 81:172-176 